
$\angle$ Research Square
Preprints are preliminary reports that have not undergone peer review.
They should not be considered conclusive, used to inform clinical practice, or referenced by the media as validated information.

\title{
Machine Learning Models with Time-Series Clinical Features to Predict Radiographic Progression in Patients with Ankylosing Spondylitis
}

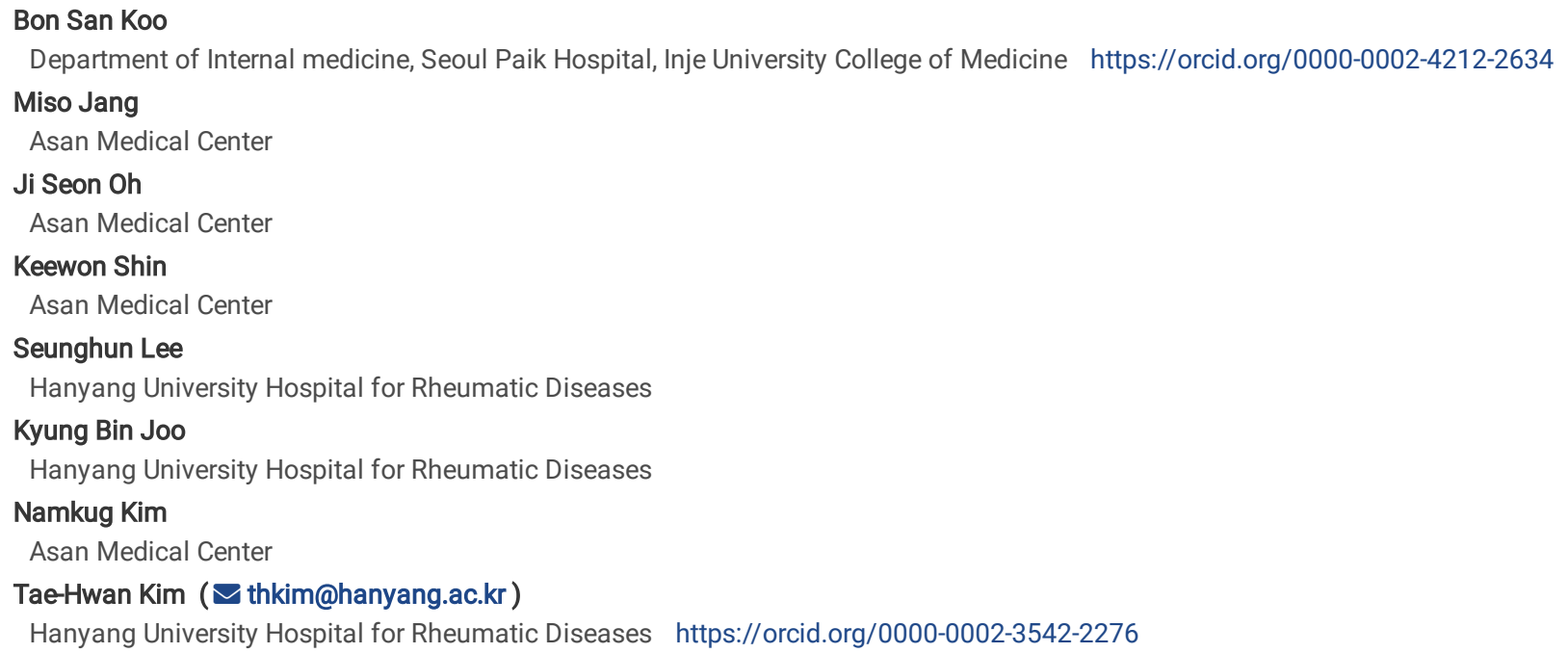




\section{Abstract}

Background: Radiographic progression in patients with ankylosing spondylitis (AS) varies between individuals, and its evaluation requires a long period of time. Previous statistical studies for radiographic progression have limitations in integrating and analyzing multiple variables of various types. The purpose of this study was to establish the application of machine learning models for predicting radiographic progression in patients with AS using time-series data from electronic medical records (EMRs).

Methods: EMR data, including baseline characteristics, laboratory finding, drug administration, and modified Stoke Ankylosing Spondylitis Spine Score (mSASSS), were collected from 1,123 AS patients who were followed up for 18 years at a common center at the time of first ( $\left.T_{1}\right)$, second $\left(T_{2}\right)$, and third ( $\left.T_{3}\right)$ visits. The radiographic progression of the

$(n+1)^{\text {th }}$ visit $\left(P_{n+1}=\left(\right.\right.$ mSASSS $_{n+1}-$ mSASSS $\left._{n}\right) /\left(T_{n+1}-T_{n}\right) \geq 1$ unit per year) was predicted using follow-up visit datasets from $T_{1}$ to $T_{n}$. Three machine learning methods (logistic regression with least absolute shrinkage and selection operation, random forest, and extreme gradient boosting algorithms) with three-fold cross validation were used.

Results: The random forest model using the $T_{1} E M R$ dataset showed the highest performance in predicting the radioactive progression $\mathrm{P}_{2}$ among all the machine learning models tested. The mean accuracy and the area under the curves were $73.73 \%$ and 0.79 , respectively. Among the variables of $\mathrm{T}_{1}$, the most important variables for predicting radiographic progression were in the order of total mSASSS, age, and alkaline phosphatase.

Conclusion: Prognosis predictive models using time-series data showed reasonable performance with clinical features of the first visit dataset for predicting radiographic progression. Additional feature data such as spine radiographs or life-log data may improve the performance of these models.

\section{Introduction}

Ankylosing spondylitis (AS) is a chronic inflammatory arthritis that primarily causes ankylosis of the spine [1]. Patients with AS have chronic inflammatory back pain and gradually develop ankylosis of the spine, limiting their movement. Because structural changes due to inflammation may potentially impact normal functioning and quality of life, the acceleration of radiographic progression is important for patients with AS.

In previous studies, statistical methods have been generally used to investigate patient features related to spinal structural change shown by radiography. They identified that radiographic progression was significantly related with men, tobacco, inflammation, and HLB-27 [2-4]. However, it is difficult to predict radiographic progression in an individual patient based on those factors because it depends on various other factors over a long period of time. In addition, there are numerous datasets in the form of electronic medical records (EMRs) for AS patients under clinical care. There may be unknown variables in these datasets contributing to radiographic progression. In the analysis and prediction of radiographic progression in AS, statistical methods may have limitations in analyzing these data. However, machine learning methods can be helpful in predicting radiographic progression using data accumulated over a long period of time and understanding the complex relationships between variables in big data.

It is difficult to clearly distinguish between statistical and machine learning models in medicine [5]. However, the strength of machine learning models is that they can handle complex and heterogeneous data such as time-series EMRs. Recently, machine learning models have been proposed to predict radiographic progression as well as disease activity, treatment response, and diagnosis [6-13]. However, these models have shown different performances owing to differences in the amount of data, tuning of hyperparameters, and outcome settings. Considering the difference in processing and outcome for data in each model, utilizing machine learning models with excellent performance to predict radiographic progression can contribute to not only saving time and healthcare cost but also improve the quality of care in clinical settings.

This study aimed to explore the application of machine learning models to predict radiographic progression in patients with AS based on time-series data for each visit and to identify predictive datasets and important features that contribute to radiographic progression in those machine learning models.

\section{Methods}

\section{Patients}

This paper describes a retrospective longitudinal study conducted at a single center. The dataset comprised reviewed EMR data of 1,280 patients between January 2001 and December 2018. All patients were previously diagnosed with AS according to the modified New York criteria [14]. The Hanyang University Seoul Hospital institutional review board approved this study (HYUH 2020-03-012-003). Informed consent was waived because this study retrospectively reviewed the EMRs.

\section{Clinical data}

Clinical characteristics including age, sex, disease duration from the first to the last follow-up, HLA-B27 positivity, eye involvement with uveitis, and peripheral joint involvement with arthritis other than axial joints were investigated. Baseline laboratory results consisted of hemoglobin ( $\mathrm{Hb})$, hematocrit (Hct), blood urea nitrogen (BUN), creatinine, aspartate transaminase (AST), alanine transaminase (ALT), alkaline phosphatase (ALP), albumin, cholesterol, protein, creatine phosphokinase (CPK), gamma glutamyl peptidase (GGT), lactate dehydrogenase (LDH), erythrocyte sedimentation rate (ESR), and C-reactive protein (CRP) levels. The prescribed drugs were classified as follows: non-steroidal anti-inflammatory drugs (NSAIDs), methotrexate, steroids, sulfasalazine, and biological disease-modifying antirheumatic drugs (bDMARDs). The mean values of laboratory tests, the sum value of prescribed medication, and clinical characteristics were used as machine learning features. 
Two radiologists (SL and KBJ) independently assessed the images and scored them according to the mSASSS (0-72) [15]. Intraobserver reliability with consistency for a reader was excellent (intraclass coefficient (ICC) $=0.978,95 \% \mathrm{Cl} 0.976$ to 0.979 ) and interobserver reliability with agreement between two readers was also excellent (ICC $=0.946,95 \% \mathrm{Cl} 0.941$ to 0.950$)[16,17]$.

\section{Model design}

The first, second, and third visits $\left(T_{1}, T_{2}\right.$, and $T_{3}$, respectively) were defined as the time points at which the first, second, and third radiographs were taken, respectively. In addition, the radiographic progression at each time point was calculated using the following formula: $P_{n+1}=\left(m_{S A S S S}+1-m S A S S S_{n}\right) /\left(T_{n+1}\right.$ $-T_{n}$ ). Radiologic progressor was defined as worsening of the mean mSASSS by more than 1 unit over 1 year, and AS patients were categorized into two groups: progressor and non-progressor [18].

We composed three clinical datasets for prediction of radiographic progression: baseline dataset at first visit $\left(\mathrm{T}_{1}\right)$ with the radiologic progression at second visit $\left(P_{2}\right)$, two-points dataset at first and second visits $\left(T_{1}+T_{2}\right)$ with the radiographic progression at third visit $\left(P_{3}\right)$, and three-points dataset at first, second, and third visit $\left(T_{1}+T_{2}+T_{3}\right)$ with radiologic progression at fourth visit $\left(P_{4}\right)$. Using three clinical dataset matrixes, we trained three prediction models for progressor and non-progressor groups (Figure 1). Three different machine learning classifiers were applied: logistic regression with least absolute shrinkage and selection operation (LASSO) using Python with the Scikit-learn package (https://github.com/scikit-learn/scikit-learn) [19], random forest (RF) using the Scikit-learn package [20], and extreme gradient boosting (XGBoost) using the Xgboost package (https://github.com/dmlc/xgboost) [21]. The algorithms were selected based on their superior performance and readiness for application. All continuous clinical features were centered and scaled to a mean of zero and a standard deviation of one (z-score transformation was performed before feature selection). The three models were run and compared to determine the best combination for determining progressor or non-progressor in the three clinical datasets. All possible combinations of hyperparameters of models were investigated by grid search using the GridSearchCV library in the Scikit-learn package [19].

With a linear combination of the selected features weighted by their respective coefficients, a LASSO regression model was used for prediction. RF, which is one of the representative ensemble methods, is widely used because it is powerful and relatively lighter than other ensemble methods. RF constructs several tree-type base models and forms an ensemble through a technique called bootstrap aggregating or bagging. XGBoost is one of the gradient boosted decision tree algorithms for large datasets. Detailed hyperparameters of the three models in the three datasets are described in the supplementary information (Supplementary eDocument 1).

\section{Performance evaluation}

The prediction models were evaluated in three rounds of three-fold cross-validation [22]. The procedures, including z-normalization and machine learning classification, were executed separately on the training data during each cross-validation. As the progressor and non-progressor groups are not equally distributed in the dataset, we used stratified cross-validation to divide the dataset. In each round, an entire dataset was randomly and equally divided into three with stratified probability. Two of these parts were used as the training dataset, and the final part was used as the test dataset. The process was repeated three times in three datasets of three models. The one-point dataset for predicting radiologic progression at second visit $\left(T_{1}\right.$ for $\left.P_{2}\right)$ had 29 features, two-point dataset for radiologic progression at third visit $\left(T_{1}+T_{2}\right.$ for $\left.P_{3}\right)$ had 53 features, and three-point dataset for radiologic progression at fourth visit ( $T_{1}+T_{2}+T_{3}$ for $\mathrm{P}_{4}$ ) had 77 features. Each average of three models of the three-fold cross-validation in the one-point dataset became the estimated performance of the models. Same was the case for the other two datasets. The predictive power of each predictor was assessed through receiver-operator characteristics (ROC).

\section{Feature selection}

Features with larger contributions to the LASSO regression model were selected. We performed feature importance analysis using RF and XGBoost to verify the robustness of the results. Variable importance was evaluated using the model-based variable importance scores and the important variables (particularly those informative to radiographic progression) were captured when the models were fitted to the training dataset $[23,24]$.

\section{Statistical analysis}

For continuous distributed data, the results are shown as means with standard deviations (SD); between-group comparisons were performed using Student's ttest. Categorical or dichotomous variables were expressed as frequencies and percentages and were compared using the chi-squared test. Area under the curves (AUCs) were used to determine the diagnostic performance, with optimal thresholds of the clinical parameters determined by maximizing the sum of the sensitivity and 1-specificity, i.e., the Youden index values. Machine learning model training and statistical analysis were performed using Python (Python Software Foundation, version 3.5.2)

\section{Results}

\section{Differences between non-progressor and progressor groups}

Of the total 1,280 patients, 157 patients did not have prescription and laboratory data; therefore, 1,123 patients were included in the study. The average time intervals between $T_{1}$ and $T_{2}$ and between $T_{2}$ and $T_{3}$ were 2.27 years and 2.12 years, respectively. The baseline characteristics of the non-progressor and progressor groups at the first visit $\left(T_{1}\right)$ are shown in Table 1. The datasets of 1,123 patients at the first visit, 1,115 patients at the second visit, and 899 patients at the third visit were divided into training and test sets. (Figure 2).

Page $3 / 9$ 


\section{Predicting radiographic progression with three time-point datasets}

The radiographic progression was predicted using the clinical data at the first, second, and third visit (Table 2). Among machine learning models, the RF model showed the best performance, which had the highest mean sensitivity, mean specificity, mean accuracy, and mean AUC than those of the lasso and logistic regression and XGBoost models. In the RF model, the prediction of radiographic progression at second visit with first visit data ( $P_{2}$ with $T_{1}$ dataset) showed better performance compared to prediction at third visit with first and second visit data $\left(P_{3}\right.$ with $T_{1}+T_{2}$ dataset) and at fourth visit with first, second, and third visit data $\left(\mathrm{P}_{4}\right.$ with $\mathrm{T}_{1}+\mathrm{T}_{2}+\mathrm{T}_{3}$ dataset).

The confusion matrix and ROC for the prediction of $\mathrm{P}_{2}$ with $\mathrm{T}_{1}$ dataset are shown in Figure $3 \mathrm{~A}$ and Figure 3B, respectively. In three-fold cross validation, the mean sensitivity, mean specificity, and mean accuracy were $73.72 \%, 73.73 \%$, and $73.73 \%$, respectively. The mean AUC of three-fold cross validation was 0.7959. (Supplementary eFigure 1 and 2 show the confusion matrix and ROC of LASSO regression and XGBoost model for $P_{2}$ with $T_{1}$ dataset, eFigure $3 A-8 A$ and eFigure 3B-8B show the confusion matrix and ROC of three machine learning models in $P_{3}$ with $T_{1}+T_{2}$ dataset and $P_{4}$ with $T_{1}+T_{2}+T_{3}$ dataset).

\section{Importance of features for predicting radiographic progression}

Variables in first visit data contributing to the prediction of radiographic progression at second visit using RF are listed in Figure $3 \mathrm{C}$. In three-fold cross validation, the most important feature in common was total mSASSS, and the second and third most important features were age and ALP followed by CRP, cholesterol, ESR, hematocrit, and ALT. Drugs such as sulfasalazine and methotrexate, clinical features such as eye and peripheral involvement, sex, and HLA B27 contributed less to radiographic progression than laboratory findings. In the XGBoost model for $\mathrm{P}_{2}$ with $\mathrm{T}_{1}$, mSASSS was the most important feature in variables; however, drugs such as sulfasalazine and methotrexate were also ranked high in importance of features (Supplementary eFigure $2 \mathrm{C}$ ). In addition, importance of features was identified in RF and XGBoost models in $\mathrm{P}_{3}$ with $\mathrm{T}_{1}+\mathrm{T}_{2}$ (Supplementary eFigure $4 \mathrm{C}$ and $5 \mathrm{C}$ ) and $\mathrm{P}_{4}$ with $\mathrm{T}_{1}+\mathrm{T}_{2}+\mathrm{T}_{3}$ (Supplementary eFigure 7C and 8C).

\section{Discussion And Conclusion}

We developed a machine learning model that predicts radiographic progression using EMR data from patients with AS accumulated over 18 years. The RF model trained on data from the first visit predicted radiographic progression after 2 years with an accuracy of $73.73 \%$ and an AUC of 0.7959 , showing the best performance among the three machine learning models. We also found that the accuracy and AUC decreased as we trained with the second and third visit data. These results suggested that the data accumulated over longer time did not increase the performance of the three machine learning models and the data from the first visit in AS may contain important predictors for predicting radiographic progression.

Joo et al. predicted radiographic progression using machine learning on the training set $(n=253)$ and test set $(n=173)$ [9]. In their results, balanced accuracy in the test set was over $65 \%$ in all models and $69.3 \%$ in RF, which was the highest of all models. In addition, the generalized linear model and support vector machine showed the best performance with an AUC of over 0.78 . The outcome of their study is similar to ours in predicting radiographic progression, but with significant differences in detail. First, we examined machine learning based prediction models for radiographic progression according to each visit using three time-point datasets containing EMR data accumulated over 18 years. We used a larger amount of time-series data compared to them and were able to identify clinical characteristics for each time point that could affect radiographic progression. These results provided various insights into radiographic progression in AS studies. In addition, the accuracy and AUC achieved in our study were higher than those achieved by them. This difference in predictive power may be related to the difference in the amount of data and variables such as limited features for bone marrow density and syndesmophyte score and additional laboratory findings.

We used time-series EMR data accumulated from the first, second, and third visits to predict radiographic progression at subsequent visits. Interestingly, the predictive performance was poorer when the model included clinical datasets at the second or third visit compared to only the clinical dataset from the first visit. This could be explained by the fact that baseline data from the first visit contain important information for predicting radiographic progression. In addition, as treatment with NSAIDs was started at the first visit, the disease activity index such as BASDAI, CRP, and ESR decreased subsequently. A decrease in the disease activity index, which affects the increase in mSASSS [2-4], may have reduced the differences in important features between individuals. As a result, the prediction performance may have deteriorated for datasets of second or third visits.

In terms of importance of features, mSASSS, age, and CRP were ranked high among the importance of features. Their association with radiographic progression is well known in statistical studies [18, 25-28]. Interestingly, among different features in our study, ALP ranked the highest in laboratory finding for predicting radiographic progression. ALP is generally produced in the liver, bone, and kidneys [29]. In serum, bone and liver specific isoform of ALP form more than $90 \%$ of total serum ALP with 1:1 ratio. In some studies, serum ALP activity was related to the inflammatory markers in mineral metabolism [30, 31]. In addition, serum ALP is associated with high disease activity, low bone mineral density, and higher structural damage scores in patients with spondyloarthritis [32]. Therefore, radiographic progression, a process of vertebral ankylosis in patients with AS, may be associated with elevated serum ALP, particularly bone-specific ALP. Statistical analysis is needed to prove the relationship between ALP and radiographic progression in future.

Several machine learning models using large datasets were useful for diagnosing axial spondyloarthritis [12]. Those approaches can help in early diagnosis and reducing the social burden of disease. Using a claim dataset, Deodhar et al. suggested that machine learning models have a positive predictive value of $6.24 \%$ compared to the Assessment of SpondyloArthritis international Society classification criteria with the positive predictive value of $1.29 \%$ [7]. In addition, machine learning models with EMR datasets have also shown good performance for predicting diagnosis of axial spondyloarthritis with accuracies ranging from $82.6 \%$ to $91.8 \%[10,11,13]$. It can be used for early diagnosis of AS by creating a machine learning model with image data as well as text data, because images such as radiographs are important in the diagnosis of AS. The detection of sacroiliitis using X-ray, computer tomography, and magnetic resonance 
images using machine learning methods has been conducted in recent years showing excellent performance in screening of patients with AS [6, 8, 33]. Therefore, developing a machine learning model useful for diagnosis by combining image, life-log, and clinical information is essential to improve diagnosis accuracy, which is worthy of future challenges for the prediction of radiographic progression in patients with AS. Furthermore, an important task is assembling a representative and diverse dataset to meet the demands of high-performance machine learning models [5].

There are some limitations to our study. First, we applied three machine learning models to predict individual radiographic progression and identified importance of features that contribute to their prediction. Interpretation of importance of features is possible since previous statistical studies have shown the factors related to radiographic progression. Therefore, machine learning methods may help complement statistical methods. However, additional statistical validation is needed for generalization of important unknown features that contribute to radiographic progression. Second, we used the EMR data from a single center. Validation using EMR data from various centers is required. Third, we utilized a machine learning model using EMR data at diagnosis and initial treatment. Therefore, this model can predict radiographic progression only when a patient first visits the hospital. In future, it is necessary to develop a model that can predict radiographic progression at various time points by advancing machine learning models.

Among datasets including first, second, and third visit, predicting the radiographic progression of the second visit using the first visit dataset showed the best performance with highest accuracy and AUC. Therefore, the clinical features of the first visit are likely to contain important information for predicting radiographic progression. In importance of features, ALP was ranked high in addition to mSASSS, age, and CRP, which are statistically related to mSASSS. In addition to EMR data, various types of data such as images and life-log may be required to increase accuracy.

\section{Declarations}

Ethics approval and consent to participate: The Hanyang University Seoul Hospital institutional review board approved this study (HYUH 2020-03-012-003). Informed consent was waived because this study retrospectively reviewed the EMRs.

Consent for publication: Not applicable.

Availability of data and material: The study protocol, statistical code, and datasets are available upon request.

Competing interests: The authors declare that they have no competing interests.

Funding: This work was supported by the National Research Foundation of Korea (NRF) grant funded by the Korea government (MSIT). (No. NRF2021R1C1C1009815)

Authors' contributions: BSK, MJ, NK, and THK contributed to the study conception and design. All authors contributed to data acquisition, analysis, or interpretation. SL and KBJ scored the spinal radiographs independently. BSK, MJ, and NK were responsible for the statistical analyses. BSK and MJ drafted the manuscript and all coauthors were involved in critical revisions for maintenance of intellectual content. NK and THK provided administrative, technical, or material support. THK had full access to all study data and takes responsibility for data integrity and data-analysis accuracy. All authors approved the final version to be submitted for publication.

Acknowledgements: We would like to thank all members of Biomedical Engineering in Asan Medical Institute of Convergence Science and Technology.

Author details: Department of Internal Medicine, Inje University Seoul Paik Hospital, Inje University College of Medicine (Koo); Department of Biomedical Engineering, Asan Medical Institute of Convergence Science and Technology, Asan Medical Center, University of Ulsan College of Medicine (Jang and Shin); Department of Medicine, University of Ulsan College of Medicine \& Asan Medical Centre (Jang); Department of Information Medicine, Big Data Research Center, Asan Medical Center (Oh); Department of Radiology, Hanyang University Hospital for Rheumatic Diseases (Lee); Department of Radiology, University of Ulsan College of Medicine, Asan Medical Center (Kim N); Department of Convergence Medicine, University of Ulsan College of Medicine \& Asan Medical Center (Kim N); Department of Rheumatology, Hanyang University Hospital for Rheumatic Diseases (Kim TH).

\section{References}

1. Inman RD: Axial Spondyloarthritis: Current Advances, Future Challenges. J Rheum Dis 2021, 28(2):55-59.

2. Brown MA, Li Z, Cao KL: Biomarker development for axial spondyloarthritis. Nat Rev Rheumatol 2020, 16(8):448-463.

3. Lorenzin M, Ometto F, Ortolan A, Felicetti M, Favero M, Doria A, Ramonda R: An update on serum biomarkers to assess axial spondyloarthritis and to guide treatment decision. Ther Adv Musculoskelet Dis 2020, 12:1759720X20934277.

4. Rademacher J, Tietz LM, Le L, Hartl A, Hermann KA, Sieper J, Mansmann U, Rudwaleit M, Poddubnyy D: Added value of biomarkers compared with clinical parameters for the prediction of radiographic spinal progression in axial spondyloarthritis. Rheumatology (Oxford) 2019, 58(9):1556-1564.

5. Rajkomar A, Dean J, Kohane I: Machine Learning in Medicine. N Engl J Med 2019, 380(14):1347-1358.

6. Bressem KK, Vahldiek JL, Adams L, Niehues SM, Haibel H, Rodriguez VR, Torgutalp M, Protopopov M, Proft F, Rademacher J et al: Deep leaming for detection of radiographic sacroiliitis: achieving expert-level performance. Arthritis Res Ther 2021, 23(1):106.

7. Deodhar A, Rozycki M, Garges C, Shukla O, Arndt T, Grabowsky T, Park Y: Use of machine leaming techniques in the development and refinement of a predictive model for early diagnosis of ankylosing spondylitis. Clin Rheumatol 2020, 39(4):975-982.

8. Faleiros MC, Nogueira-Barbosa MH, Dalto VF, Junior JRF, Tenorio APM, Luppino-Assad R, Louzada-Junior P, Rangayyan RM, de Azevedo-Marques PM: Machine learning techniques for computer-aided classification of active inflammatory sacroiliitis in magnetic resonance imaging. Adv Rheumato/2020, 
60(1):25

9. Joo YB, Baek IW, Park YJ, Park KS, Kim KJ: Machine learning-based prediction of radiographic progression in patients with axial spondyloarthritis. Clin Rheumatol 2020, 39(4):983-991.

10. Walsh JA, Pei S, Penmetsa G, Hansen JL, Cannon GW, Clegg DO, Sauer BC: Identification of Axial Spondyloarthritis Patients in a Large Dataset: The Development and Validation of Novel Methods. J Rheumato/ 2020, 47(1):42-49.

11. Walsh JA, Pei S, Penmetsa GK, Leng J, Cannon GW, Clegg DO, Sauer BC: Cohort identification of axial spondyloarthritis in a large healthcare dataset: current and future methods. BMC Musculoskelet Disord 2018, 19(1):317.

12. Walsh JA, Rozycki M, Yi E, Park Y: Application of machine learning in the diagnosis of axial spondyloarthritis. Curr Opin Rheumatol2019, 31(4):362-367.

13. Walsh JA, Shao Y, Leng J, He T, Teng CC, Redd D, Treitler Zeng Q, Burningham Z, Clegg DO, Sauer BC: Identifying Axial Spondyloarthritis in Electronic Medical Records of US Veterans. Arthritis Care Res (Hoboken) 2017, 69(9):1414-1420.

14. van der Linden S, Valkenburg HA, Cats A: Evaluation of diagnostic criteria for ankylosing spondylitis. A proposal for modification of the New York criteria. Arthritis Rheum 1984, 27(4):361-368.

15. Creemers MC, Franssen MJ, van't Hof MA, Gribnau FW, van de Putte LB, van Riel PL: Assessment of outcome in ankylosing spondylitis: an extended radiographic scoring system. Ann Rheum Dis 2005, 64(1):127-129.

16. Koo BS, Oh JS, Park SY, Shin JH, Ahn GY, Lee S, Joo KB, Kim TH: Tumour necrosis factor inhibitors slow radiographic progression in patients with ankylosing spondylitis: 18-year real-world evidence. Ann Rheum Dis 2020, 79(10):1327-1332.

17. Lee TH, Koo BS, Nam B, Oh JS, Park SY, Lee S, Joo KB, Kim TH: Conventional disease-modifying antinheumatic drugs therapy may not slow spinal radiographic progression in ankylosing spondylitis: results from an 18-year longitudinal dataset. Ther Adv Musculoskelet Dis 2020, 12:1759720X20975912.

18. Haroon N, Inman RD, Learch TJ, Weisman MH, Lee M, Rahbar MH, Ward MM, Reveille JD, Gensler LS: The impact of tumor necrosis factor alpha inhibitors on radiographic progression in ankylosing spondylitis. Arthritis Rheum 2013, 65(10):2645-2654.

19. Pedregosa F, Varoquaux G, Gramfort A, Michel V, Thirion B, Grisel O, Blondel M, Prettenhofer P, Weiss R, Dubourg V et al: Scikit-learn: Machine Learning in Python. Journal of Machine Learning Research 2012, 12.

20. Breiman L: Random Forests. Machine Learning 2001, 45(1):5-32.

21. Sheridan RP, Wang M, Liaw A, Ma J, Gifford E: Correction to Extreme Gradient Boosting as a Method for Quantitative Structure-Activity Relationships. Journal of Chemical Information and Modeling 2020, 60(3):1910-1910.

22. Kim J-H: Estimating classification error rate: Repeated cross-validation, repeated hold-out and bootstrap. Computational Statistics \& Data Analysis 2009, 53(11):3735-3745.

23. Greenwell BM, Boehmke BC, McCarthy AJ: A Simple and Effective Model-Based Variable Importance Measure. ArXiv 2018, abs/1805.04755.

24. Kuhn M, Johnson K: Applied Predictive Modeling; 2013.

25. Poddubnyy D, Haibel H, Listing J, Marker-Hermann E, Zeidler H, Braun J, Sieper J, Rudwaleit M: Baseline radiographic damage, elevated acute-phase reactant levels, and cigarette smoking status predict spinal radiographic progression in early axial spondylarthritis. Arthritis Rheum 2012, 64(5):13881398.

26. Poddubnyy DA, Rudwaleit M, Listing J, Braun J, Sieper J: Comparison of a high sensitivity and standard C reactive protein measurement in patients with ankylosing spondylitis and non-radiographic axial spondyloarthritis. Ann Rheum Dis 2010, 69(7):1338-1341.

27. Ramiro S, van der Heijde D, van Tubergen A, Stolwijk C, Dougados M, van den Bosch F, Landewe R: Higher disease activity leads to more structural damage in the spine in ankylosing spondylitis: 12-year longitudinal data from the OASIS cohort. Ann Rheum Dis 2014, 73(8):1455-1461.

28. Poddubnyy D, Protopopov M, Haibel H, Braun J, Rudwaleit M, Sieper J: High disease activity according to the Ankylosing Spondylitis Disease Activity Score is associated with accelerated radiographic spinal progression in patients with early axial spondyloarthritis: results from the GErman SPondyloarthritis Inception Cohort. Ann Rheum Dis 2016, 75(12):2114-2118.

29. Haarhaus M, Brandenburg V, Kalantar-Zadeh K, Stenvinkel P, Magnusson P: Alkaline phosphatase: a novel treatment target for cardiovascular disease in CKD. Nat Rev Nephrol 2017, 13(7):429-442.

30. Cheung BMY, Ong KL, Cheung RV, Wong LYF, Wat NMS, Tam S, Leung GM, Cheng CH, Woo J, Janus ED et al: Association between plasma alkaline phosphatase and C-reactive protein in Hong Kong Chinese. Clinical Chemistry and Laboratory Medicine 2008, 46(4):523-527.

31. Damera S, Raphael KL, Baird BC, Cheung AK, Greene T, Beddhu S: Serum alkaline phosphatase levels associate with elevated serum C-reactive protein in chronic kidney disease. Kidney Int 2011, 79(2):228-233.

32. Kang KY, Hong YS, Park S-H, Ju JH: Increased serum alkaline phosphatase levels correlate with high disease activity and low bone mineral density in patients with axial spondyloarthritis. Seminars in Arthritis and Rheumatism 2015, 45(2):202-207.

33. Castro-Zunti R, Park EH, Choi Y, Jin GY, Ko SB: Early detection of ankylosing spondylitis using texture features and statistical machine learning, and deep learning, with some patient age analysis. Comput Med Imaging Graph 2020, 82:101718.

\section{Tables}

Table 1. Baseline characteristics in patients with non-progression and progression. 


\begin{tabular}{|c|c|c|c|c|}
\hline Variables & Total patients $(\mathrm{n}=1123)$ & Non-progressor $(n=830)$ & Progressor $(n=293)$ & $\mathrm{P}$ \\
\hline Male, n (\%) & $993(88.42)$ & $718(86.51)$ & $275(93.86)$ & 0.001 \\
\hline Age (years) & $32.01 \pm 9.41$ & $30.98 \pm 9.46$ & $34.93 \pm 8.65$ & $<0.001$ \\
\hline Eye involvement, $\mathrm{n}(\%)$ & $363(32.32)$ & $245(29.53)$ & $118(40.27)$ & $<0.001$ \\
\hline Peripheral involvement, $\mathrm{n}(\%)$ & $401(35.71)$ & $319(38.43)$ & $82(27.99)$ & 0.002 \\
\hline HLA-B27, n (\%) & 1079 (96.08) & $793(95.54)$ & $286(97.61)$ & 0.163 \\
\hline ALP & $79.51 \pm 32.98$ & $77.82 \pm 32.16$ & $84.28 \pm 34.82$ & 0.005 \\
\hline ALT & $21.55 \pm 16.64$ & $21.06 \pm 16.91$ & $22.95 \pm 15.81$ & 0.084 \\
\hline AST & $19.96 \pm 9.24$ & $19.94 \pm 9.36$ & $20.00 \pm 8.89$ & 0.921 \\
\hline Albumin & $4.33 \pm 1.07$ & $4.38 \pm 1.01$ & $4.19 \pm 1.21$ & 0.019 \\
\hline BUN & $12.94 \pm 4.69$ & $13.14 \pm 4.54$ & $12.37 \pm 5.06$ & 0.022 \\
\hline CPK & $96.40 \pm 231.63$ & $99.21 \pm 243.98$ & $88.43 \pm 192.56$ & 0.444 \\
\hline CRP & $1.74 \pm 2.09$ & $1.61 \pm 2.00$ & $2.10 \pm 2.31$ & 0.001 \\
\hline Cholesterol & $162.48 \pm 50.70$ & $162.31 \pm 48.81$ & $162.94 \pm 55.79$ & 0.864 \\
\hline Creatinine & $0.83 \pm 0.31$ & $0.83 \pm 0.22$ & $0.83 \pm 0.48$ & 0.999 \\
\hline ESR & $28.57 \pm 27.30$ & $26.89 \pm 26.93$ & $33.32 \pm 27.82$ & $<0.001$ \\
\hline GGT & $14.97 \pm 30.47$ & $13.52 \pm 26.00$ & $19.09 \pm 40.31$ & 0.028 \\
\hline $\mathrm{Hb}$ & $13.40 \pm 3.17$ & $13.46 \pm 3.02$ & $13.24 \pm 3.57$ & 0.346 \\
\hline Hct & $40.64 \pm 9.36$ & $40.78 \pm 8.88$ & $40.23 \pm 10.60$ & 0.427 \\
\hline LDH & $114.39 \pm 77.68$ & $115.65 \pm 77.13$ & $110.81 \pm 79.23$ & 0.366 \\
\hline NSAIDs, n (\%) & $880(78.36)$ & $650(78.31)$ & $230(78.50)$ & 0.987 \\
\hline bDMARDs, n (\%) & $246(21.91)$ & 185 (22.29) & $61(20.82)$ & 0.659 \\
\hline Methotrexate, n (\%) & $151(13.45)$ & $122(14.70)$ & $29(9.90)$ & 0.049 \\
\hline steroids, n (\%) & $260(23.15)$ & $197(23.73)$ & $63(21.50)$ & 0.485 \\
\hline sulfasalazine, n (\%) & $283(25.20)$ & $228(27.47)$ & $55(18.77)$ & 0.004 \\
\hline mSASSS & $14.57 \pm 16.28$ & $12.36 \pm 16.07$ & $20.84 \pm 15.25$ & $<0.001$ \\
\hline
\end{tabular}

HLA, human leukocyte antigen; ALP, Alkaline phosphatase; AST, aspartate aminotransferase; ALT, alanine aminotransferase; BUN, Blood Urea Nitrogen; CPK, creatine phosphokinase; CRP, C-reactive protein; ESR, erythrocyte sedimentation rate; GGT, gamma glutamyl peptidase; Hb, hemoglobin; Hct, hematocrit; LDH, Lactate Dehydrogenase; NSAIDs, nonsteroidal anti-inflammatory drugs; bDMARDs, biologic disease modifying anti-rheumatic drugs; mSASSS, the modified Stoke Ankylosing Spondylitis Spinal Score.

Table 2. Prediction performance evaluation according to time points and machine learning models.

\begin{tabular}{|c|c|c|c|c|c|c|c|c|c|c|c|}
\hline \multirow{2}{*}{$\begin{array}{l}\text { Prediction } \\
\text { of } \\
\text { radiographic } \\
\text { progression } \\
(P n+1) \text { with } \\
\text { visit data } \\
(\mathrm{Tn})\end{array}$} & \multicolumn{4}{|c|}{ Lasso and logistic regression } & \multicolumn{4}{|c|}{ Random forest } & \multicolumn{3}{|l|}{ XGBoost } \\
\hline & $\begin{array}{l}\text { Sensitivity } \\
\text { (\%) }\end{array}$ & $\begin{array}{l}\text { Specificity } \\
(\%)\end{array}$ & $\begin{array}{l}\text { Accuracy } \\
(\%)\end{array}$ & AUC & $\begin{array}{l}\text { Sensitivity } \\
(\%)\end{array}$ & $\begin{array}{l}\text { Specificity } \\
(\%)\end{array}$ & $\begin{array}{l}\text { Accuracy } \\
(\%)\end{array}$ & AUC & $\begin{array}{l}\text { Sensitivity } \\
(\%)\end{array}$ & $\begin{array}{l}\text { Specificity } \\
(\%)\end{array}$ & $\begin{array}{l}\text { Accuracy } \\
(\%)\end{array}$ \\
\hline P2 with T1 & 68.25 & 68.31 & 68.3 & 0.7169 & 73.72 & 73.73 & 73.73 & 0.7959 & 70.99 & 70.84 & 70.88 \\
\hline $\begin{array}{l}\text { P3 with } \\
\text { T1+T2 }\end{array}$ & 66.18 & 66.3 & 66.27 & 0.6831 & 67.95 & 67.27 & 67.44 & 0.7467 & 66.21 & 66.3 & 66.28 \\
\hline $\begin{array}{l}\text { P4 with } \\
\mathrm{T} 1+\mathrm{T} 2+\mathrm{T} 3\end{array}$ & 61.39 & 60.03 & 60.4 & 0.6442 & 68.47 & 67.93 & 68.08 & 0.7348 & 66.8 & 67.94 & 67.63 \\
\hline
\end{tabular}

AUC, area under curve

\section{Figures}


Radiographic progression $\left(P_{n+1}\right):\left(\right.$ mSASSS $_{n+1}-$ mSASSS $\left._{n}\right) \div\left(T_{n+1}-T_{n}\right)$
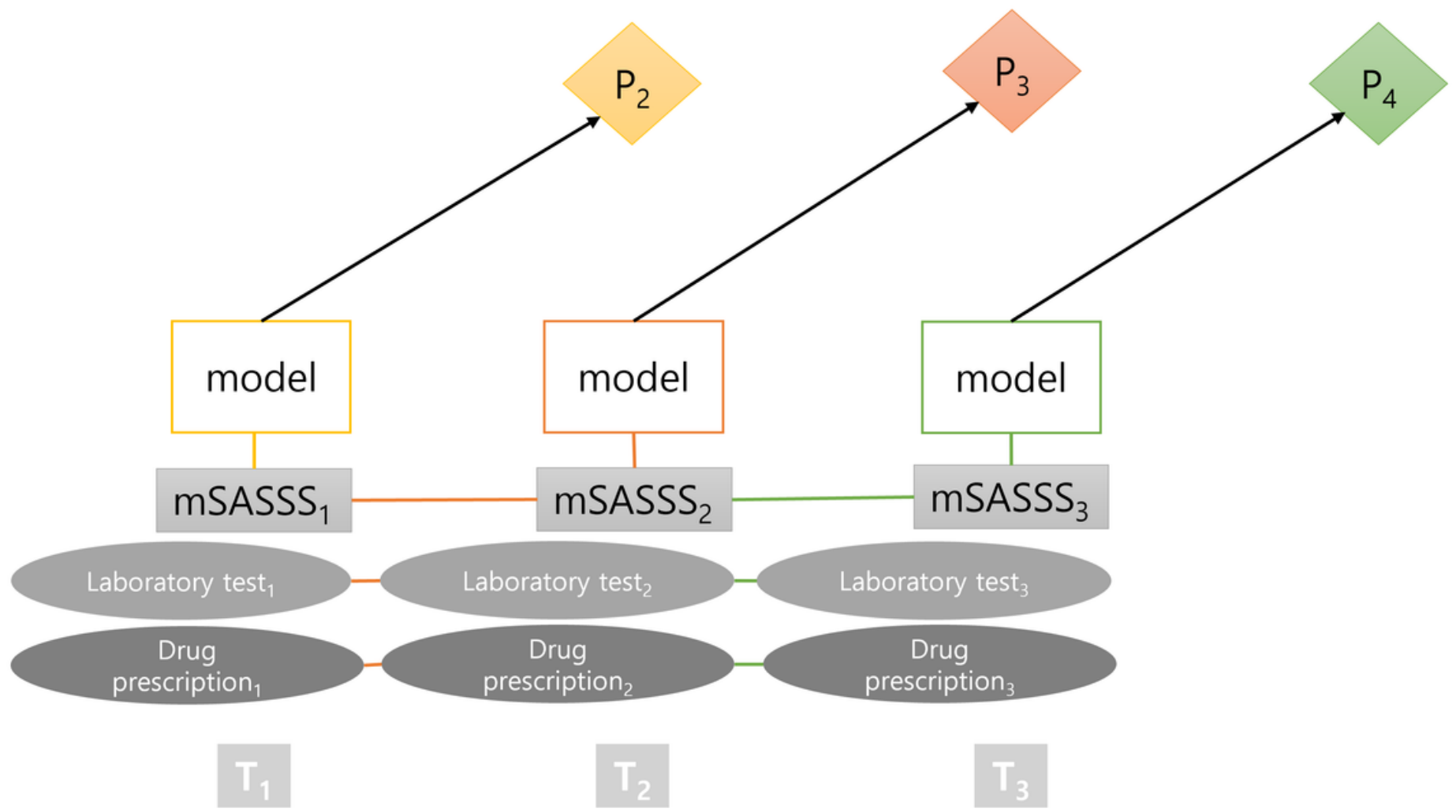

Figure 1

Time points for prediction of radiographic progression. Dataset including clinical information of first, second, and third visit was T1, T2, and T3, respectively. Radiographic progression of second, third, fourth visit was P2, P3, and P4.

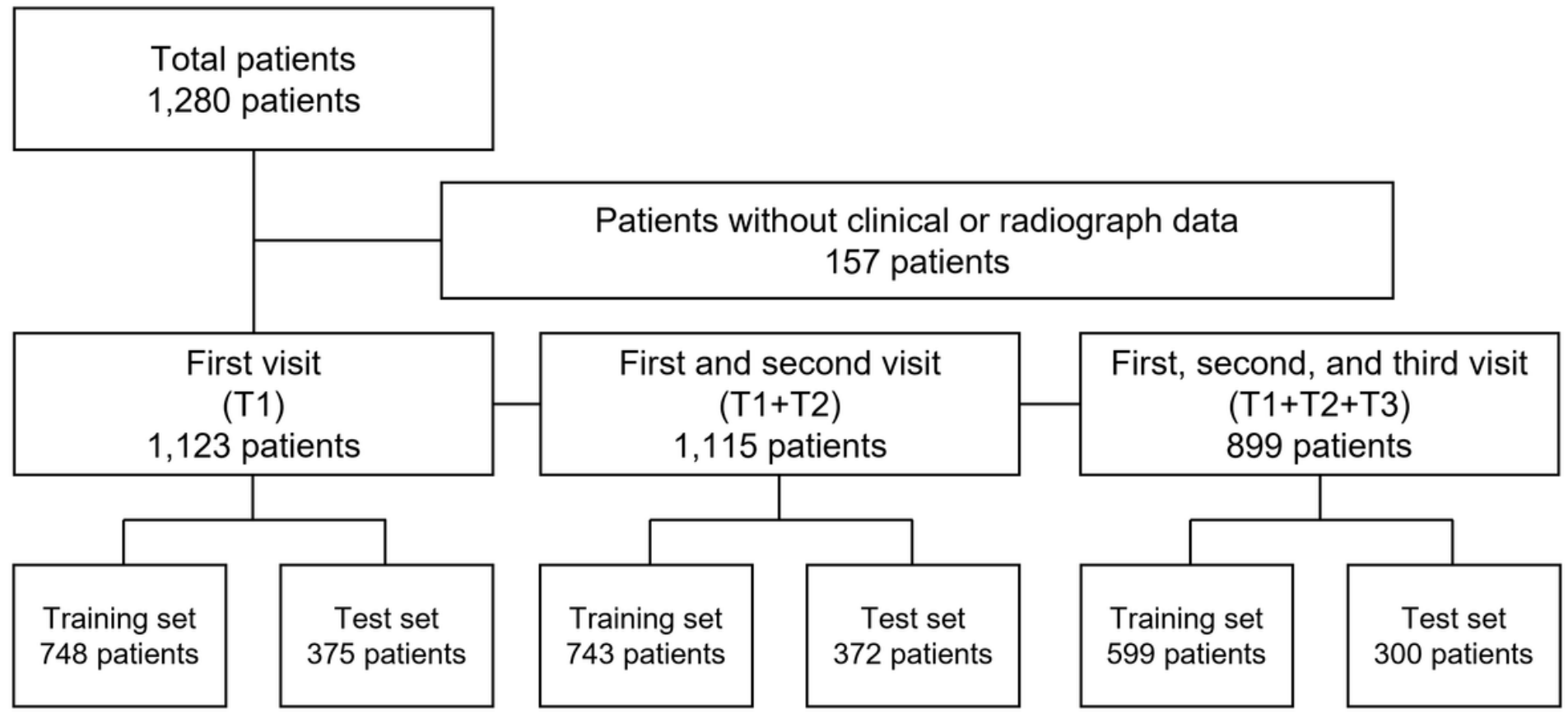


A chart flow of the study.

A
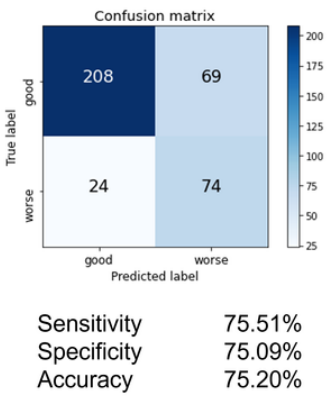

1-fold cross validation

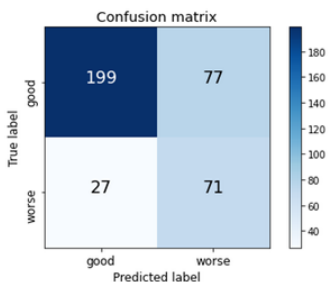

Sensitivity $\quad 72.45 \%$

Specificity $\quad 72.10 \%$

Accuracy $\quad 72.19 \%$

2-fold cross validation

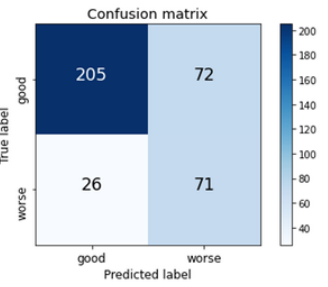

Sensitivity $\quad 73.20 \%$

Specificity $\quad 74.01 \%$

Accuracy $\quad 73.80 \%$

3-fold cross validation

B

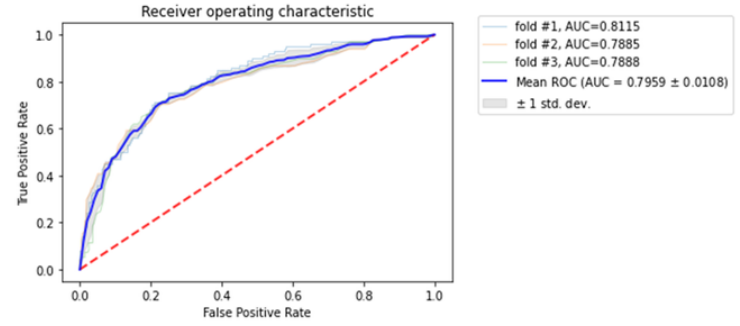

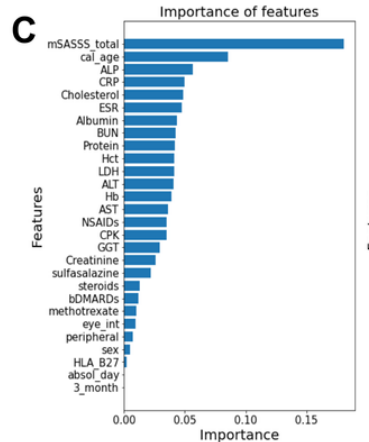

1 -fold cross validation

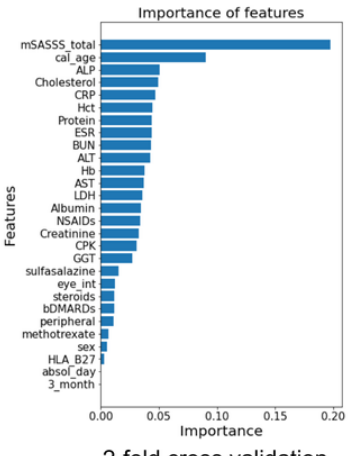

2-fold cross validation

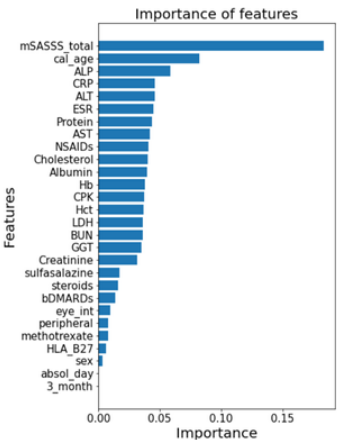

3-fold cross validation

\section{Figure 3}

Prediction in random forest model. Confusion matrix (A), AUROC (B) and importance of features in cross validation (C). AUROC: The area under the receiver operating characteristic.

\section{Supplementary Files}

This is a list of supplementary files associated with this preprint. Click to download.

- 20210922supplFigures.docx 